\title{
Evidence of increased brain amyloid in severe TBI survivors at 1, 12, and 24 months after injury: report of 2 cases
}

\author{
Joshua W. Gatson, PhD, ${ }^{1,2}$ Cari Stebbins, MPH, ${ }^{1}$ Dana Mathews, MD, ${ }^{3}$ Thomas S. Harris, MSBME, ${ }^{4}$ \\ Christopher Madden, MD, ${ }^{2}$ Hunt Batjer, MD, ${ }^{2}$ Ramon Diaz-Arrastia, MD, ${ }^{5}$ and Joseph P. Minei, MD' \\ Departments of ${ }^{1}$ Surgery, ${ }^{2}$ Neurological Surgery, ${ }^{3}$ Radiology, and ${ }^{4}$ Neurology, University of Texas Southwestern Medical Center, \\ Dallas, Texas; and ${ }^{5}$ Center for Neuroscience and Regenerative Medicine, Uniformed Services University of the Health Sciences, \\ Rockville, Maryland
}

\begin{abstract}
Traumatic brain injury (TBI) is a major risk factor for Alzheimer's disease. With respect to amyloid deposition, there are no published serial data regarding the deposition rate of amyloid throughout the brain after TBI. The authors conducted serial ${ }^{18} \mathrm{~F}-\mathrm{AV}-45$ (florbetapir F18) positron emission tomography (PET) imaging in 2 patients with severe TBI at 1, 12, and 24 months after injury. A total of 12 brain regions were surveyed for changes in amyloid levels.

Case 1 involved a 50-year-old man who experienced a severe TBI. Compared with the 1-month time point, of the 12 brain regions that were surveyed, a decrease in amyloid (as indicated by standard uptake value ratios) was only observed in the hippocampus (-16\%, left; $-12 \%$, right) and caudate nucleus $(-18 \%$, left; $-18 \%$, right), suggesting that initial amyloid accumulation in the brain was cleared between time points 1 and 12 months after injury. Compared to the scan at 1 year, a greater increase in amyloid $(+15 \%)$ was observed in the right hippocampus at the 24 -month time point. The patient in Case 2 was a 37-year-old man who suffered severe trauma to the head and a subsequent stroke; he had poor cognitive/functional outcomes and underwent 1.5 years of rehabilitation. Due to a large infarct area on the injured side of the brain (right side), the authors focused primarily on brain regions affected within the left hemisphere. Compared with the 1-month scan, they only found an increase in brain amyloid within the left anterior putamen (+11\%) at 12 months after injury. In contrast, decreased amyloid burden was detected in the left caudate nucleus (-48\%), occipital cortex (-21\%), and precuneus $(-19 \%)$ brain regions at the 12-month time point, which is indicative of early accumulation and subsequent clearance. In comparison with 12-month values, more clearance was observed, since a reduction in amyloid was found at 24 months after trauma within the left anterior putamen $(-12 \%)$ and occipital cortex $(-15 \%)$. Also, by 24 months, most of the amyloid had been cleared and the patient demonstrated improved results on the Rivermead symptom questionnaire, Glasgow Outcome Scale-Extended, and Disability Rating Scale. With respect to APOE status, the patient in Case 1 had two $\varepsilon 3$ alleles and the patient in Case 2 had one $\varepsilon 2$ and one $\varepsilon 3$ allele.

In comparison to the findings of the initial scan at 1 month after TBI, by 12 and 24 months after injury amyloid was cleared in some brain regions and increased in others. Serial imaging conducted here suggests that florbetapir F18 PET imaging may be useful in monitoring amyloid dynamics within specific brain regions following severe TBI and may be predictive of cognitive deficits.
\end{abstract}

http://thejns.org/doi/abs/10.3171/2015.6.JNS15639

KEY WORDS amyloid; florbetapir F18; traumatic brain injury

$\mathrm{E}$ ACH year in the United States alone, about 300,000 people are hospitalized for traumatic brain injury (TBI), with a mortality rate of $16 \% .^{1,26}$ After TBI, secondary brain injury escalates due in part to heightened levels of oxidant injury, inflammation, and amyloid production. Increases in secondary brain injury may begin almost immediately after the primary injury ${ }^{8,15}$ and evolve into chronic neurodegenerative conditions.

TBI is a major risk factor for the development of chronic neurodegenerative conditions such as Alzheimer's disease (AD). $4,11,18,20,29$ Postmortem analyses of brains from TBI patients have revealed that the degree of amyloid plaque

ABBREVIATIONS AD = Alzheimer's disease; DRS = Disability Rating Scale; GCS = Glasgow Coma Scale; GOS-E = Glasgow Outcome Scale-Extended; MMSE = MiniMental State Examination; MNI = Montreal Neurological Institute; SUVR = standard uptake value ratio; TBI = traumatic brain injury; VOI = volume of interest.

SUBMITTED March 21, 2015. ACCEPTED June 4, 2015.

INCLUDE WHEN CITING Published online November 27, 2015; DOI: 10.3171/2015.6.JNS15639. 
deposition in the brain correlated with the level of cognitive impairment. ${ }^{11,16,18,20}$ Also, several autopsy studies have shown amyloid plaques are present in the brain as early as 2 hours after injury and persist even 2.5 years later. ${ }^{11,23,30}$ Furthermore, the likelihood of amyloid plaque deposition was more evident in patients with $A P O E \& 4$ expression (50\% in Apo-E4-expressing patients vs $30 \%$ in the nonApo-E4-expressing group). The Apo-E4-expressing individuals also performed worse on cognitive tests at time points more distant from the injury. ${ }^{4,19}$ Given the increased risk for AD noted in Apo-E4-expressing patients, the increased likelihood for amyloid plaque deposition in TBI patients with the APOE $\varepsilon 4$ allele supports the reported link between severe TBI and AD-like dementia. ${ }^{19}$ In this study, we hypothesized that people who express Apo-E4 will accumulate more amyloid in the brain after TBI.

Recently, a single PET scan ([11C] PiB) of the head conducted within 1 year of TBI showed that amyloid deposition correlates with histological findings of amyloid plaques in the brain of people who died shortly after the lone scan. ${ }^{10}$ In this study we used ${ }^{18} \mathrm{~F}-\mathrm{AV}-45$ (florbetapir F18), which has a longer half-life and has a high binding affinity for fibrillar amyloid, 3,6 as a more practical tool for imaging the head of TBI survivors. In previous AD studies, florbetapir F18 binding was characterized and found to correlate with cognitive and mood disorders. . $^{3,6,12,17,35}$

In this prospective study, the aim was to conduct serial imaging in TBI survivors to determine the deposition profile within a number of brain regions at subacute $(\sim 30$ days after injury) and chronic (12 and 24 months) time points after injury.

\section{Case Reports}

\section{Case 1}

History

This 50-year-old man had experienced a severe TBI (Glasgow Coma Scale [GCS] score of 3) due to a motor vehicle collision (Table 1). CT scans showed that he had a 3-mm midline shift. Additionally, a subdural hematoma was reported in the left frontal and temporoparietal cortex. To lower intracranial pressure, a left hemicraniectomy was performed (Fig. 1A and B).

\section{Serial Florbetapir F18 PET Imaging}

Following discharge from Parkland Hospital (Dallas, Texas) the patient underwent scanning with the Siemens ECAT HR PET scanner on Day 30 after the injury, and

TABLE 1. Characteristics of patients with severe TBI

\begin{tabular}{lcc}
\hline Characteristic & Case 1 & Case 2 \\
\hline Age & 50 & 37 \\
\hline Sex & Male & Male \\
\hline GCS score & 3 & 4 \\
\hline CT findings & SDH & SAH \\
\hline LOS & 30 & 60 \\
\hline APOE status & $\varepsilon 3 / \varepsilon 3$ & $\varepsilon 2 / \varepsilon 3$ \\
\hline
\end{tabular}

LOS = length of hospital stay; $\mathrm{SAH}=$ subarachnoid hematoma; $\mathrm{SDH}=$ subdural hematoma.
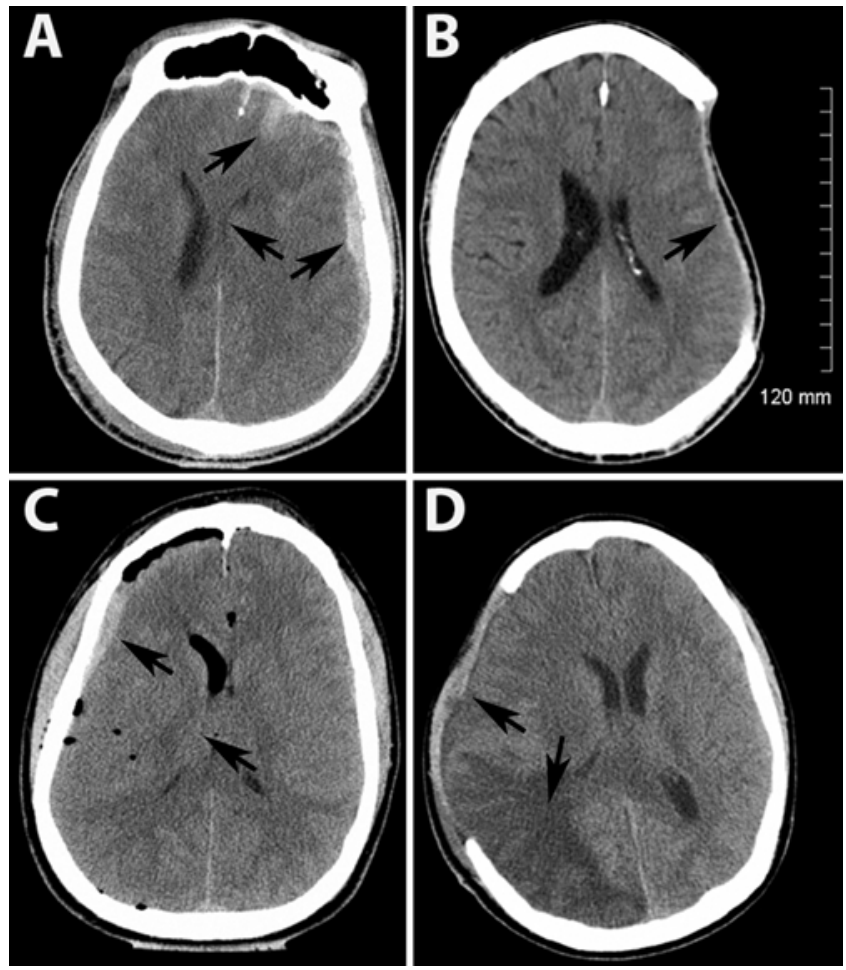

FIG. 1. CT scans obtained in Case 1 (A, within the first 6 hours after injury; B, postcraniotomy) and Case 2 (C, within the first 6 hours after injury; D, postcraniotomy). Arrows indicate locations of hematomas, ventricles, infarct areas, and craniotomy.

subsequent scanning was performed at 1 and 2 years after injury. To label the fibrillar amyloid, $370 \mathrm{MBq}(10 \mathrm{mCi})$ of the florbetapir F18 tracer (AVID Radiopharmaceuticals) was injected. Following a 50-minute washout period, a 10-minute continuous brain PET scan was conducted. Images were reconstructed immediately after the 10-minute scan. We acquired individual measures of amyloid deposition for specific brain structures. A hierarchical regression technique was used to analyze the data. Images were spatially normalized to the Montreal Neurological Institute (MNI) space using SPM8 and special image templates developed for florbetapir F18 from the brains of normal controls. These values were used to extract a value of amyloid from the data. A negative scan was defined as one in which the majority of the tracer was bound in the white matter (Fig. 2A and B). Initially, the scans were analyzed for significant accumulation of tracer in the gray matter. A scan is considered positive if tracer retention is evident primarily in the cortical gray areas (Fig. 2C). After spatial normalization, volumes of interest (VOIs) that were derived from MRI data from the MNI atlas were applied using internally developed MATLAB code to extract count density from 12 brain regions (anterior and posterior cingulate gyrus, dorsolateral prefrontal cortex, frontal cortex, left and right hippocampus, left and right anterior putamen, left and right caudate nucleus, left and right thalamus, occipital cortex, orbital frontal cortex, parietal cortex, precuneus, temporal cortex). Standard uptake value ratios (SUVRs) were computed for VOIs and a cerebellar VOI was extracted to determine nonspecific binding. As 

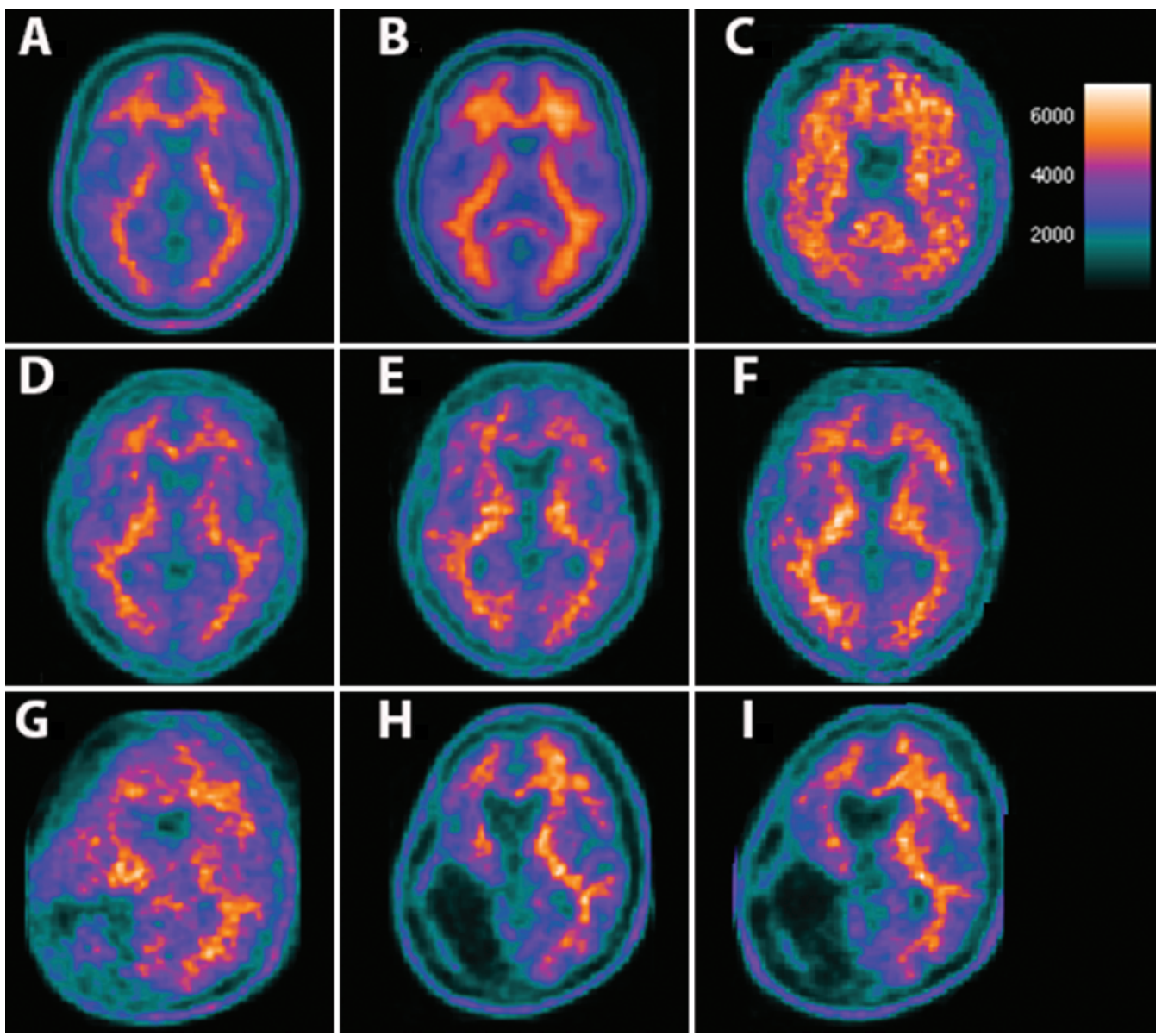

FIG. 2. Florbetapir F18 PET images. Transverse sections of florbetapir F18 PET scans obtained in healthy age-matched controls (A, 50-year-old control for Case 1; B, 38-year-old control for Case 2); the patient in Case 1 at 1, 12, and 24 months after injury (D, $\mathbf{E}$, and $\mathbf{F}$, respectively); and the patient in Case 2 at 1, 12, and 24 months after injury ( $\mathbf{G}, \mathbf{H}$, and $\mathbf{I}$, respectively). Panel $\mathbf{C}$ is a positive control for cortical uptake of florbetapir F18. Figure is available in color online only.

determined previously in healthy controls who underwent serial scanning performed by our research group and others, ${ }^{24}$ a change in SUVRs greater than $8 \%$ per year was deemed to be a meaningful increase. All aspects of the study described herein were approved by the institutional review board at the University of Texas Southwestern Medical Center (Dallas, Texas) prior to the initiation of any study-specific procedures.

\section{APOE Genotyping}

To test for apolipoprotein E (APOE) status, venous blood samples $(8 \mathrm{ml})$ were collected into EDTA tubes, aliquoted into $1-\mathrm{ml}$ tubes, and immediately frozen at $-80^{\circ} \mathrm{C}$. Genomic DNA was isolated by standard protocols. Genotypes were determined by Taqman assays (Applied Biosystems, Inc.).

\section{Results}

Prior to the scanning procedures, the Mini-Mental State Examination (MMSE), the Disability Rating Scale (DRS), and the Rivermead Post-Concussion Symptom Questionnaire $^{13}$ were administered. The patient had a moderate-to-severe cognitive impairment, as indicated by an MMSE score of 19 at the 1-month time point. He also had a moderately severe disability (DRS score of 9). Scores of 29 and 4 for the MMSE and DRS (moderate), respectively, indicated improved cognition and function at the 12-month time point. Also, the patient had a lower good recovery as indicated by a Glasgow Outcome ScaleExtended (GOS-E) score of 7 at Year 1. In contrast, at the 24-month time point, a worsening of both cognition (MMSE score of 22) and function (DRS score of 7) was observed (Table 2). At 1 month after injury, the patient displayed severe symptoms, which improved slightly at the 12- (moderate) and 24- (moderate) month time points (Table 3). He suffered from headaches, nausea, balance, dizziness, fatigue, and memory problems (Table 3 ). The results of $A P O E$ genotyping showed that the patient's status was $A P O E \varepsilon 3 / \varepsilon 3$. Comparison of the patient's florbetapir F18 PET scans from all imaging time points to scans obtained in a healthy age-matched control did not show any gross accumulation of amyloid within the cortical gray matter (Fig. 2), and thus analysis of amyloid levels within specific brain regions was performed. SUVR values from the 12 brain regions are listed in Table 4. In the patient in Case 1, comparison of region-specific SUVRs at 1 month with the 12-month scan only showed a decrease in amyloid in the hippocampus and caudate nucleus regions. Within the 
TABLE 2. Outcomes of severe TBI

\begin{tabular}{ccc}
\hline Outcome & Case 1 & Case 2 \\
\hline MMSE & & 23 \\
\hline 1 mo & 19 & 29 \\
\hline 12 mos & 29 & 29 \\
\hline 24 mos & 22 & \\
\hline GOS-E & & 6 \\
\hline 12 mos & 7 & 7 \\
\hline 24 mos & 7 & 15 \\
\hline DRS & 9 & 9 \\
\hline 1 mo & 9 & 5 \\
\hline 12 mos & 7 &
\end{tabular}

hippocampus, $16 \%$ and $12 \%$ decreases in amyloid were observed in the left and right hippocampus, respectively, at Year 1. A similar finding was observed in the left $(-18 \%)$ and right $(-18 \%)$ caudate nucleus. A decrease in SUVR at the 12-month time point signifies amyloid clearance from the brain since the initial scan. In the caudate nucleus and left hippocampal brain regions, no change in amyloid deposition was observed at the 24-month time point. In contrast, a $15 \%$ increase of amyloid was found in the right hippocampus at the 24-month time point compared with the SUVR values collected at 12 months (Table 4, Fig. 3). With respect to post-TBI symptoms, worsening in memory problems (Rivermead symptom questionnaire) and cognitive outcomes (MMSE) was also observed at the 24-month time point compared with results obtained at 12 months (Tables 2 and 3).

\section{Case 2}

History

This 37-year-old man was involved in a motor vehicle collision and was admitted to Parkland Hospital with a severe TBI (GCS score of 4). A 1- to 2-mm midline shift and subarachnoid hemorrhage in the right frontotemporoparietal cortex were identified by CT. A hemicraniectomy was performed to reduce intracranial pressure in the right hemisphere. This patient also suffered a right hemispheric

TABLE 3. Rivermead symptom scoring for the patient in Case 1 at 1,12 , and 24 months after TBI

\begin{tabular}{lccc}
\hline \multicolumn{1}{c}{ Symptom } & 1 Mo & 12 Mos & 24 Mos \\
\hline Headaches & 4 & 4 & 3 \\
\hline Nausea \&/or vomiting & 3 & 2 & 1 \\
\hline Feelings of dizziness & 4 & 3 & 2 \\
\hline $\begin{array}{l}\text { Fatigue, tiring more } \\
\text { easily }\end{array}$ & 4 & 3 & 3 \\
\hline $\begin{array}{l}\text { Forgetfulness, poor } \\
\text { memory }\end{array}$ & 4 & 2 & 4 \\
\hline $\begin{array}{l}\text { Average symptom } \\
\text { score }\end{array}$ & 3.8 (severe) & 2.8 (moderate) & 2.6 (moderate) \\
\hline
\end{tabular}

\footnotetext{
* Symptom rating: no problem, 0-1.5; mild, 1.6-2.5; moderate, 2.6-3.5;
} severe, 3.6-4.
TABLE 4. SUVRs for 1,12 , and 24 months after TBI in Case 1 *

\begin{tabular}{|c|c|c|c|}
\hline Brain Region & $1 \mathrm{Mo}$ & 12 Mos & 24 Mos \\
\hline \multicolumn{4}{|l|}{ Cingulate gyrus } \\
\hline Anterior cingulate gyrus & 1.17 & 1.07 & 1.19 \\
\hline Posterior cingulate gyrus & 1.18 & 1.27 & 1.30 \\
\hline Dorsolateral prefrontal cortex & 1.18 & 1.08 & 1.14 \\
\hline Frontal cortex & 1.14 & 1.04 & 1.09 \\
\hline \multicolumn{4}{|l|}{ Hippocampus } \\
\hline Left hippocampus & 0.99 & $0.84(-16 \%)$ & 0.84 \\
\hline Right hippocampus & 1.07 & $0.96(-12 \%)$ & $1.10(+15 \%)$ \\
\hline \multicolumn{4}{|l|}{ Anterior putamen } \\
\hline Left anterior putamen & 1.21 & 1.31 & 1.38 \\
\hline Right anterior putamen & 1.32 & 1.28 & 1.31 \\
\hline \multicolumn{4}{|l|}{ Caudate nucleus } \\
\hline Left caudate nucleus & 0.94 & $0.81(-18 \%)$ & 0.86 \\
\hline Right caudate nucleus & 0.96 & $0.83(-18 \%)$ & 0.84 \\
\hline \multicolumn{4}{|l|}{ Thalamus } \\
\hline Left thalamus & 1.29 & 1.21 & 1.15 \\
\hline Right thalamus & 1.19 & 1.14 & 1.17 \\
\hline Occipital cortex & 1.11 & 1.09 & 1.08 \\
\hline Orbital frontal cortex & 1.08 & 0.97 & 0.96 \\
\hline Parietal cortex & 1.30 & 1.22 & 1.29 \\
\hline Precuneus & 1.22 & 1.22 & 1.29 \\
\hline Temporal cortex & 1.23 & 1.17 & 1.21 \\
\hline
\end{tabular}

* The values in bold within the 1-, 12-, and 24-month columns are higher than expected ( $5 \%-8 \%$ increase per year) when compared to the previous time point.

ischemic stroke during his hospital stay (Fig. 1C and D). He subsequently underwent 1.5 years of rehabilitation to improve swallowing, walking, and other motor abilities.

\section{Serial Florbetapir F18 PET Imaging}

Scanning was performed at 1,12 , and 24 months after TBI using the Siemens ECAT HR PET scanner. At each time point, the patient was injected with the florbetapir F18 tracer and a brain scan was conducted. Images were processed as described in Case 1, and 12 brain regions were surveyed to collect SUVRs to ascertain if changes in amyloid deposition occurred in a particular brain region.

\section{APOE Genotyping}

As in Case 1 , venous blood samples $(8 \mathrm{ml})$ were collected into EDTA tubes, aliquoted into $1 \mathrm{ml}$ tubes, and immediately frozen at $-80^{\circ} \mathrm{C}$. Genomic DNA was isolated by standard protocols. Genotypes were determined by Taqman assays.

\section{Results}

At the initial scan ( 1 month after injury), the patient had a DRS rating of 15 (severe disability), an MMSE score of 23 (moderate cognitive deficit), and Rivermead symptom scores indicating severe symptoms. At the later time points, the DRS score improved to 9 and 5 at the 12- and 24-month time points, respectively. The MMSE score improved to 29 at both 12 and 24 months. At Year 1, the 


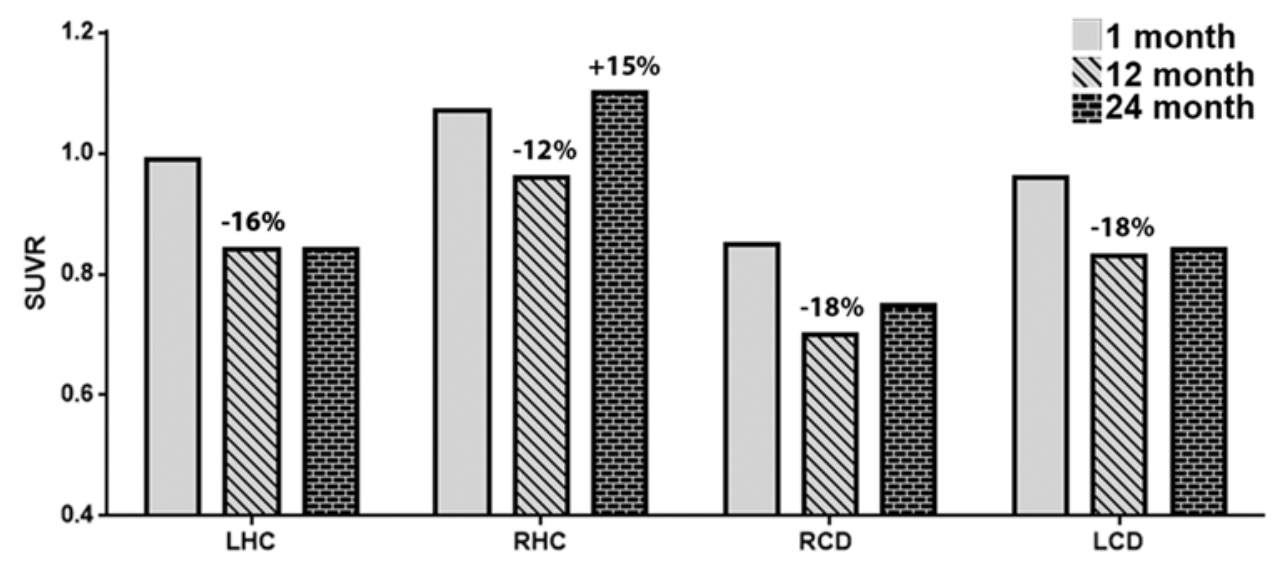

FIG. 3. Case 1: percent change of SUVR at 12 and 24 months after injury. Of the 12 brain regions surveyed, differences were observed in the left and right hippocampus (LHC, RHC) and caudate (LCD, RCD) brain regions.

patient had an upper moderate disability and Rivermead symptom scoring indicated severe symptoms. Improvement of symptom scoring to moderate and GOS-E scoring to low-good recovery was observed at 24 months. The symptoms reported for this patient were headaches, nausea, vomiting, balance, dizziness, fatigue, irritability, sadness, and memory problems (Table 5). Compared with the agematched control, no evidence of a global increase of florbetapir F18 was found within the cortical tissue at the 1-, 12-, or 24-month time points (Fig. 2G-I). Due to the infarct area in the right hemisphere and possible data reliability issues, we only focused on the SUVR values from brain regions outside of the affected area. In the left hemisphere, compared with the 1-month scan, we observed an increase in amyloid at 12 months in the left anterior putamen $(+11 \%)$ and a decrease in the left caudate nucleus $(-48 \%)$, occipital cortex $(-21 \%)$, and precuneus $(-19 \%)$ brain regions. No increase in brain amyloid was observed at 24 months in any of the regions that were surveyed. Within the left anterior putamen $(-12 \%)$, occipital cortex $(-15 \%)$, and precuneus $(-11 \%)$, compared with 12 months, a decrease in amyloid was measured at 24 months after TBI (Table 6, Fig. 4). The $A P O E$ genotype for this patient was APOE $\varepsilon 2 / \varepsilon 3$.

TABLE 5. Rivermead symptom scoring for the patient in Case 2 at 1,12 , and 24 months after TBI*

\begin{tabular}{lccc}
\hline \multicolumn{1}{c}{ Symptom } & 1 Mo & 12 Mos & 24 Mos \\
\hline Headaches & 4 & 4 & 3 \\
\hline Nausea \&/or vomiting & 4 & 4 & 2 \\
\hline Feelings of dizziness & 4 & 4 & 3 \\
\hline $\begin{array}{l}\text { Fatigue, tiring more } \\
\text { easily }\end{array}$ & 4 & 4 & 4 \\
\hline $\begin{array}{l}\text { Feeling frustrated or } \\
\text { impatient }\end{array}$ & 3 & 3 & 2 \\
$\begin{array}{l}\text { Forgetfulness, poor } \\
\text { memory }\end{array}$ & 4 & 3 & 2 \\
\hline $\begin{array}{l}\text { Average symptom } \\
\text { score }\end{array}$ & 3.8 (severe) & 3.7 (severe) & 2.6 (moderate) \\
\hline
\end{tabular}

* Symptom rating: no problem, 0-1.5; mild, 1.6-2.5; moderate, 2.6-3.5; severe, 3.6-4.

\section{Discussion}

In this study, we demonstrate the feasibility of obtaining serial scans in people who have survived a severe TBI event. Also, we show that in our sample of 2 TBI patients, brain scans obtained in the patient with the poorer outcome showed more amyloid accumulation than scans obtained in the patient with the better outcome or in healthy controls. The novelty of this study is in its conduct of se-

TABLE 6. SUVRs for 1,12 , and 24 Months after TBI: Case $2^{*}$

\begin{tabular}{|c|c|c|c|}
\hline Brain Region & $1 \mathrm{Mo}$ & 12 Mos & 24 Mos \\
\hline \multicolumn{4}{|l|}{ Cingulate gyrus } \\
\hline Anterior cingulate gyrus & 1.15 & 1.06 & 1.09 \\
\hline Posterior cingulate gyrus & 1.14 & 1.16 & 1.05 \\
\hline Dorsolateral prefrontal cortex & 0.92 & 0.97 & 0.91 \\
\hline Frontal cortex & 0.94 & 0.90 & 0.86 \\
\hline \multicolumn{4}{|l|}{ Hippocampus } \\
\hline Left hippocampus & 1.01 & 1.03 & 1.12 \\
\hline Right hippocampus & 0.75 & 0.23 & 0.23 \\
\hline \multicolumn{4}{|l|}{ Anterior putamen } \\
\hline Left anterior putamen & 1.08 & $1.23(+11 \%)$ & $1.09(-12 \%)$ \\
\hline Right anterior putamen & 0.91 & 1.39 & 1.37 \\
\hline \multicolumn{4}{|l|}{ Caudate nucleus } \\
\hline Left caudate nucleus & 0.76 & $0.37(-48 \%)$ & 0.32 \\
\hline Right caudate nucleus & 0.74 & 0.74 & 0.71 \\
\hline \multicolumn{4}{|l|}{ Thalamus } \\
\hline Left thalamus & 0.97 & 0.88 & 0.85 \\
\hline Right thalamus & 1.31 & 0.78 & 0.54 \\
\hline Occipital cortex & 0.79 & $0.62(-21 \%)$ & $0.53(-15 \%)$ \\
\hline Orbital frontal cortex & 0.96 & 1.08 & 1.06 \\
\hline Parietal cortex & 0.82 & 0.81 & 0.71 \\
\hline Precuneus & 1.01 & $0.82(-19 \%)$ & $0.73(-11 \%)$ \\
\hline Temporal cortex & 0.94 & 0.91 & 0.81 \\
\hline
\end{tabular}

* Bolded brain regions from the right hemisphere were not included in subsequent analysis due to an infarct in the right hemisphere. The values in bold within the 1-, 12-, and 24-month columns are higher than expected (5\%-8\% increase per year) when compared to the previous time point. 


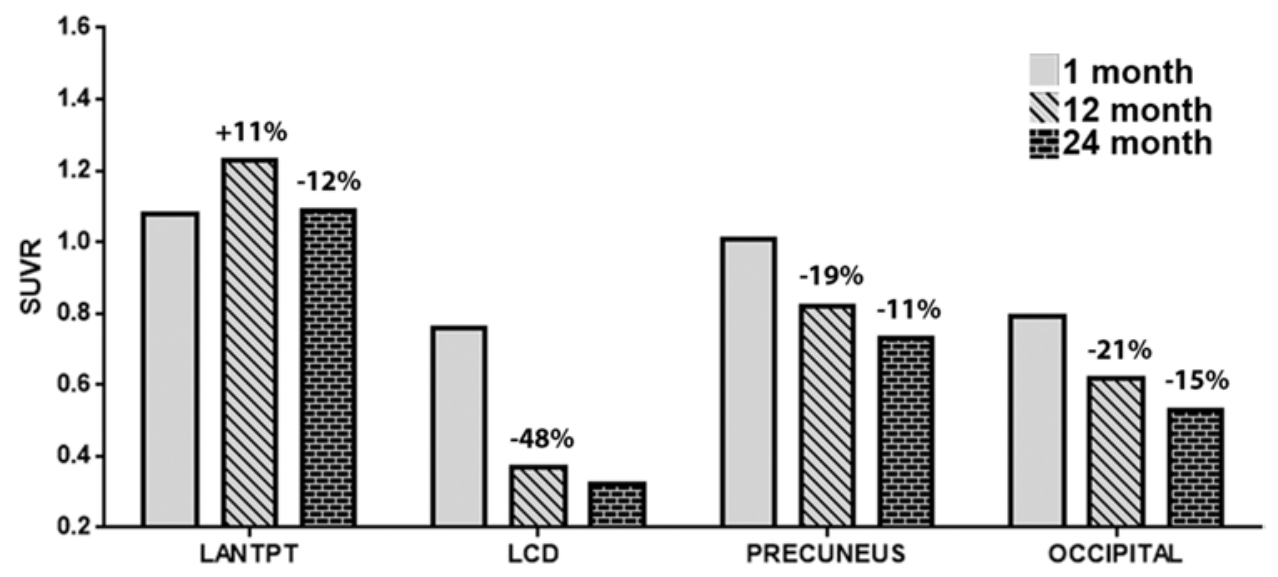

FIG. 4. Case 2: percent change of SUVR at 12 and 24 months after injury. There were distinct changes in the 12-and 24-month SUVRs in the left anterior putamen (LANTPT), left caudate (LCD), precuneus, and occipital cortex brain regions compared with the 1-month time point

rial scanning to compare the subacute $(\sim 30$ days after TBI) amyloid levels to levels demonstrated by subsequent scans performed at 12 and 24 months after injury to determine the rate of change of amyloid in the brain within each person. By conducting serial scans, we can elucidate whether increases in amyloid deposition predict worsening of amyloid accumulation and/or neurodegeneration. Also, this methodology sheds light on the dynamics of amyloid deposition rates and patterns during the acute and chronic phases of TBI. Use of florbetapir F18, which has a substantial half-life (90 minutes), was instrumental in identifying both clearance and accumulation of amyloid at 12 and 24 months after injury when compared with SUVRs collected during the initial scan $(\sim 1$ month after injury) within each subject. When surveying specific brain regions, we found that both of the patients experienced increases in brain amyloid after TBI (Figs. 3 and 4). Originally, we hypothesized that accumulation of amyloid in TBI survivors only occurs in individuals with poor outcomes. In contrast, as defined by the participants' DRS and GOS-E scores, we found that TBI patients with both moderate-to-severe and moderate-to-good outcomes exhibited increases in amyloid deposition in the brain. A main difference that we observed is that the patient with a moderate-to-good outcome (Case 1) exhibited a lower SUVR percent change (mean 15\%) than the patient in Case 2, who experienced a moderate-to-poor outcome and a mean change of amyloid of about $26 \%$ at the 12-month time point. These differences may be related to the initial severity of their injuries.

To determine region-specific changes, we decided to compare the SUVRs between the 3 time points to determine if fluctuations exist throughout cortical and subcortical structures. This approach proved to be valuable in comparing the changes in brain amyloid within these serial scans. As shown in Tables 4 and 6, only a small number of brain regions were affected within each individual. Use of florbetapir F18 as a clinical marker for brain regions with heightened amyloid load may prove useful in predicting the onset of long-term cognitive and mood disorders. For example, the patient in Case 1 had an initial increase in amyloid in the left and right hippocampi that was reduced by 12 months after TBI. In contrast to the 12 -month scan, the 24-month scan showed a greater elevation of amyloid in the right hippocampus. This change may be directly related to the onset of severe memory problems, which was reported at the 24-month appointment by the patient in Case 1 and his family. Comparison of the SUVR values from multiple time points within a single patient may yield more reliable data on the timing of amyloid deposition after TBI. If the individuals who may be prone to accumulate amyloid can be identified, anti-amyloid therapies that become available in the future may provide an ideal treatment to reduce amyloid-related chronic secondary brain injury.

In previous studies, the likelihood of amyloid plaque deposition was more evident in TBI patients with Apo-E4 expression (50\% in Apo-E4-expressing patients vs 30\% in the non-Apo-E4-expressing group). The Apo-E4-expressing individuals also performed worse on cognitive tests at longer periods of time after injury. ${ }^{2,4,5,9,14,19,21,22,28,33,34}$ Given the increased risk for AD noted in Apo-E4-expressing patients, the increased likelihood for amyloid plaque deposition in $A P O E \& 4$ carriers with TBI supports the reported link between severe TBI and AD. 4,7, ,14,19,22,25,27,28,31-34 Initially, we hypothesized that only Apo-E4 expressers would accumulate amyloid in the brain after TBI. Unfortunately, due to our limited sample size, we were unable to test our hypothesis. In our 2 cases, the patients' APOE genotypes were as follows: $A P O E \varepsilon 3 / \varepsilon 3$ in Case 1 and $A P O E \varepsilon 2 / \varepsilon 3$ in Case 2 (Table 1).

There are several recognized limitations of this study. Primarily, the limited sample size, lack of controls (trauma without TBI), variability of the injuries, and diverse pattern of amyloid accumulation between the participants limit the conclusions that can be drawn. However, the primary purpose of this study was to assess a serial imaging methodology. Using purposive sampling, we enrolled TBI patients who had improvements in neurological outcomes and were most likely to survive and participate in followup procedures, since the severe TBI population suffers from high morbidity and low follow-up. As a future direction, we plan to enroll TBI patients with both poor and good outcomes to determine if amyloid deposition rates differ between individuals with good outcomes and those 
with poor outcomes. With respect to the diversity of amyloid deposition in the brain, the difference observed here may be due to the mode and location of the injury, which is highly variable within the TBI population. We also recognize that administration of an extensive neuropsychological battery would prove useful to determine relationships between amyloid deposition and cognitive/mood deficits. In addition to neuropsychological testing, in future definitive studies, we will also conduct diffusion tensor imaging and functional connectivity MRI to measure the integrity of the white matter within these populations.

In conclusion, this research sheds light on the use of florbetapir F18 PET imaging as a method to detect/monitor amyloid accumulation in the brain of TBI survivors. We found that within a 2-year period after severe TBI, amyloid accumulates at both early and late postinjury time points. By conducting follow-up scans, we were able to identify specific brain regions where amyloid accumulated after TBI as potential biomarkers for cognitive and/ or mood deficits. As a future direction, administration of a thorough neuropsychological battery and MRI may prove useful in determining how amyloid deposition in certain brain structures correlates with cognitive performance and structural abnormalities after TBI at both the early and late time points within each individual.

\section{Acknowledgments}

The florbetapir F18 radiotracer was kindly provided by AVID Radiopharmaceuticals for imaging conducted at 1 and 12 months after injury. AVID Radiopharmaceuticals also provided agematched control florbetapir F18 scans. We would like to thank Dr. Ryan Huebinger and Mrs. Ling-yu Chang, who conducted the $A P O E$ genotyping experiments. We also acknowledge Drs. DaiWai Olson, Meharvan Singh, and James Simpkins, who assisted with editing the manuscript.

\section{References}

1. Adamides AA, Winter CD, Lewis PM, Cooper DJ, Kossmann T, Rosenfeld JV: Current controversies in the management of patients with severe traumatic brain injury. ANZ J Surg 76:163-174, 2006

2. Alexander S, Kerr ME, Kim Y, Kamboh MI, Beers SR, Conley YP: Apolipoprotein E4 allele presence and functional outcome after severe traumatic brain injury. J Neurotrauma 24:790-797, 2007

3. Choi SR, Golding G, Zhuang Z, Zhang W, Lim N, Hefti F, et al: Preclinical properties of ${ }^{18} \mathrm{~F}-\mathrm{AV}-45$ : a PET agent for $\mathrm{A} \beta$ plaques in the brain. J Nucl Med 50:1887-1894, 2009

4. DeKosky ST, Abrahamson EE, Ciallella JR, Paljug WR, Wisniewski SR, Clark RS, et al: Association of increased cortical soluble A $\beta 42$ levels with diffuse plaques after severe brain injury in humans. Arch Neurol 64:541-544, 2007

5. Diaz-Arrastia R, Baxter VK: Genetic factors in outcome after traumatic brain injury: what the human genome project can teach us about brain trauma. J Head Trauma Rehabil 21:361-374, 2006

6. Fleisher AS, Chen K, Liu X, Roontiva A, Thiyyagura P, Ayutyanont N, et al: Using positron emission tomography and florbetapir F18 to image cortical amyloid in patients with mild cognitive impairment or dementia due to Alzheimer disease. Arch Neurol 68:1404-1411, 2011

7. Fleminger S, Oliver DL, Lovestone S, Rabe-Hesketh S, Giora A: Head injury as a risk factor for Alzheimer's disease: the evidence 10 years on; a partial replication. J Neurol Neurosurg Psychiatry 74:857-862, 2003

8. Gatson JW, Warren V, Abdelfattah K, Wolf S, Hynan LS, Moore $C$, et al: Detection of $\beta$-amyloid oligomers as a predictor of neurological outcome after brain injury. J Neurosurg 118:1336-1342, 2013

9. Guo Z, Cupples LA, Kurz A, Auerbach SH, Volicer L, Chui $\mathrm{H}$, et al: Head injury and the risk of AD in the MIRAGE study. Neurology 54:1316-1323, 2000

10. Hong YT, Veenith T, Dewar D, Outtrim JG, Mani V, Williams C, et al: Amyloid imaging with carbon 11-labeled Pittsburgh compound B for traumatic brain injury. JAMA Neurol 71:23-31, 2014

11. Ikonomovic MD, Uryu K, Abrahamson EE, Ciallella JR, Trojanowski JQ, Lee VM, et al: Alzheimer's pathology in human temporal cortex surgically excised after severe brain injury. Exp Neurol 190:192-203, 2004

12. Johnson KA, Minoshima S, Bohnen NI, Donohoe KJ, Foster NL, Herscovitch P, et al: Appropriate use criteria for amyloid PET: a report of the Amyloid Imaging Task Force, the Society of Nuclear Medicine and Molecular Imaging, and the Alzheimer's Association. Alzheimers Dement 9:e-1-e-16, 2013

13. King NS, Crawford S, Wenden FJ, Moss NE, Wade DT: The Rivermead Post Concussion Symptoms Questionnaire: a measure of symptoms commonly experienced after head injury and its reliability. J Neurol 242:587-592, 1995

14. Liberman JN, Stewart WF, Wesnes K, Troncoso J: Apolipoprotein E epsilon 4 and short-term recovery from predominantly mild brain injury. Neurology 58:1038-1044, 2002

15. MacKenzie JD, Siddiqi F, Babb JS, Bagley LJ, Mannon LJ, Sinson GP, et al: Brain atrophy in mild or moderate traumatic brain injury: a longitudinal quantitative analysis. AJNR Am J Neuroradiol 23:1509-1515, 2002

16. Marklund N, Farrokhnia N, Hånell A, Vanmechelen E, Enblad P, Zetterberg H, et al: Monitoring of $\beta$-amyloid dynamics after human traumatic brain injury. J Neurotrauma 31:42-55, 2014

17. Mattsson N, Tosun D, Insel PS, Simonson A, Jack CR Jr, Beckett LA, et al: Association of brain amyloid- $\beta$ with cerebral perfusion and structure in Alzheimer's disease and mild cognitive impairment. Brain 137:1550-1561, 2014

18. Mortimer JA, French LR, Hutton JT, Schuman LM: Head injury as a risk factor for Alzheimer's disease. Neurology 35:264-267, 1985

19. Nicoll JA, Roberts GW, Graham DI: Apolipoprotein E epsilon 4 allele is associated with deposition of amyloid betaprotein following head injury. Nat Med 1:135-137, 1995

20. Plassman BL, Havlik RJ, Steffens DC, Helms MJ, Newman TN, Drosdick D, et al: Documented head injury in early adulthood and risk of Alzheimer's disease and other dementias. Neurology 55:1158-1166, 2000

21. Ponsford J, McLaren A, Schönberger M, Burke R, Rudzki D, Olver J, et al: The association between apolipoprotein E and traumatic brain injury severity and functional outcome in a rehabilitation sample. J Neurotrauma 28:1683-1692, 2011

22. Rebeck GW, Reiter JS, Strickland DK, Hyman BT: Apolipoprotein E in sporadic Alzheimer's disease: allelic variation and receptor interactions. Neuron 11:575-580, 1993

23. Roberts GW, Allsop D, Bruton C: The occult aftermath of boxing. J Neurol Neurosurg Psychiatry 53:373-378, 1990

24. Rodrigue KM, Kennedy KM, Devous MD Sr, Rieck JR, Hebrank AC, Diaz-Arrastia R, et al: $\beta$-Amyloid burden in healthy aging: regional distribution and cognitive consequences. Neurology 78:387-395, 2012

25. Roses AD: Apolipoprotein E genotyping in the differential diagnosis, not prediction, of Alzheimer's disease. Ann Neurol 38:6-14, 1995

26. Sahuquillo J, Arikan F: Decompressive craniectomy for the 
treatment of refractory high intracranial pressure in traumatic brain injury. Cochrane Database Syst Rev (1):CD003983, 2006

27. Salib E, Hillier V: Head injury and the risk of Alzheimer's disease: a case control study. Int J Geriatr Psychiatry 12:363-368, 1997

28. Schmechel DE, Saunders AM, Strittmatter WJ, Crain BJ, Hulette CM, Joo SH, et al: Increased amyloid $\beta$-peptide deposition in cerebral cortex as a consequence of apolipoprotein $\mathrm{E}$ genotype in late-onset Alzheimer disease. Proc Natl Acad Sci U S A 90:9649-9653, 1993

29. Simpkins JW: Commentary on "Alzheimer's Disease Drug Development and the Problem of the Blood-Brain Barrier". Alzheimers Dement 5:433-434, 2009

30. Smith DH, Chen XH, Iwata A, Graham DI: Amyloid $\beta$ accumulation in axons after traumatic brain injury in humans. J Neurosurg 98:1072-1077, 2003

31. Sorbi S, Nacmias B, Piacentini S, Repice A, Latorraca S, Forleo P, et al: ApoE as a prognostic factor for post-traumatic coma. Nat Med 1:852, 1995 (Letter)

32. Teasdale GM, Nicoll JA, Murray G, Fiddes M: Association of apolipoprotein E polymorphism with outcome after head injury. Lancet 350:1069-1071, 1997

33. Tiraboschi P, Hansen LA, Masliah E, Alford M, Thal LJ, Corey-Bloom J: Impact of APOE genotype on neuropathologic and neurochemical markers of Alzheimer disease. Neurology 62:1977-1983, 2004

34. Wisniewski T, Frangione B: Apolipoprotein E: a pathological chaperone protein in patients with cerebral and systemic amyloid. Neurosci Lett 135:235-238, 1992
35. Wu KY, Hsiao IT, Chen CS, Chen CH, Hsieh CJ, Wai YY, et al: Increased brain amyloid deposition in patients with a lifetime history of major depression: evidenced on ${ }^{18} \mathrm{~F}$-florbetapir (AV-45/Amyvid) positron emission tomography. Eur J Nucl Med Mol Imaging 41:714-722, 2014

\section{Disclosures}

The authors report no conflict of interest concerning the materials or methods used in this study or the findings specified in this paper.

\section{Author Contributions}

Conception and design: Gatson, Mathews, Diaz-Arrastia, Minei. Acquisition of data: Gatson, Stebbins, Mathews. Analysis and interpretation of data: Gatson, Harris, Madden, Diaz-Arrastia. Drafting the article: Gatson, Diaz-Arrastia, Minei. Critically revising the article: Gatson, Mathews, Madden, Batjer, DiazArrastia, Minei. Reviewed submitted version of manuscript: all authors. Approved the final version of the manuscript on behalf of all authors: Gatson. Administrative/technical/material support: Stebbins, Mathews. Study supervision: Gatson, Mathews, Madden, Batjer, Minei.

\section{Correspondence}

Joshua W. Gatson, Department of Surgery, Department of Neurosurgery, UT Southwestern Medical Center, 5323 Harry Hines Blvd., Dallas, TX 75390-9160. email: joshua.gatson@utsw.edu. 